\title{
Parameter estimation of linear and quadratic chirps by employing the fractional fourier transform and a generalized time frequency transform
}

\author{
SHISHIR B SAHAY ${ }^{1}$, T MEGHASYAM ${ }^{2, *}$, RAHUL K ROY ${ }^{3}$, \\ GAURAV POONIWALA ${ }^{2}$, SASANK CHILAMKURTHY ${ }^{2}$ and \\ VIKRAM GADRE ${ }^{2}$ \\ ${ }^{1}$ Military Institute of Technology, Girinagar, Pune 411025 , India \\ ${ }^{2}$ Indian Institute of Technology Bombay, Mumbai 400 076, India \\ ${ }^{3}$ INS Tunir, Mumbai 400 704, India \\ e-mail: sbsahay1@yahoo.com; itismeghasyam@gmail.com; \\ royrahul764@gmail.com; gtpooniwala@gmail.com; \\ sasankchilamkurthy@gmail.com; vmgadre@ee.iitb.ac.in
}

MS received 10 April 2014; revised 8 January 2015; accepted 23 January 2015

\begin{abstract}
This paper is targeted towards a general readership in signal processing. It intends to provide a brief tutorial exposure to the Fractional Fourier Transform, followed by a report on experiments performed by the authors on a Generalized Time Frequency Transform (GTFT) proposed by them in an earlier paper. The paper also discusses the extension of the uncertainty principle to the GTFT. This paper discusses some analytical results of the GTFT. We identify the eigenfunctions and eigenvalues of the GTFT. The time shift property of the GTFT is discussed. The paper describes methods for estimation of parameters of individual chirp signals on receipt of a noisy mixture of chirps. A priori knowledge of the nature of chirp signals in the mixture - linear or quadratic is required, as the two proposed methods fall in the category of model-dependent methods for chirp parameter estimation.
\end{abstract}

Keywords. Mixture of chirp signals; time-frequency plane; instantaneous frequency (IF); model-dependent methods for chirp parameter estimation; Fractional Fourier Transform (FrFT); Generalized Time-Frequency Transform (GTFT); Uncertainty principle; Eigenfunction.

*For correspondence 


\section{Introduction}

Efficient methods for analysis and parameter estimation of chirp signals are becoming increasingly important in sonar, geophysical, bio-medical and radar applications. The analytic form of the general chirp signal is represented by the following equation:

$$
\begin{gathered}
c(t)=e^{j \cdot \phi(t)} \\
f(t)=\frac{1}{2 \pi} \cdot \frac{\mathrm{d} \phi(t)}{\mathrm{d} t} .
\end{gathered}
$$

As seen in Eq. (2), the instantaneous frequency $f(t)$ is a function of time $t$. If a linear relationship exists between frequency and time, the chirp is known as a linear frequency modulated (LFM) chirp. A quadratic relationship between frequency and time results in a quadratic frequency modulated (QFM) or quadratic chirp signal. More esoteric relationships between frequency and time result in different kinds of chirp signals. Signals of the form $\sin (\phi(t))$ or $\cos (\phi(t))$ are also known as chirp signals.

In contrast to chirps, in case of the ordinary complex exponential signal $e^{j 2 \pi f_{0} t}$, here $f_{0}$ is a constant and the ordinary sinusoidal signal $\cos \left(2 \pi f_{0} t\right)$ and $\sin \left(2 \pi f_{0} t\right)$, the rate of change of phase and consequently the IF is constant throughout the duration of the signal. A LFM chirp and a QFM chirp are shown in figure 1. Note the linear and quadratic relationship respectively between IF and time in the two cases shown in the figure.

Parameter estimation of chirp signals normally involves estimation of the unknown relationship between IF and time.

Various methods are discussed in literature for detection and parameter estimation of the chirp signals in a noisy environment. Some methods are model-independent i.e. they require no a priori knowledge of nature of the chirp signal. Some examples include filter bank approach (Sahay et al 2012b), particle swarm optimization. While some methods are model-dependent i.e. they require a priori knowledge about the nature of the chirp signal - linear or quadratic relationship between the time and instantaneous frequency. Most of them use techniques like Fractional Fourier Transform (FrFT), Wigner Ville Distribution (WVD), Discrete Cosine Transform (DCT) (Chassande-Mottin \& Flandrin 1999; Gal et al. 2011; Djuric \& Kay 1990; Ma Min et al. 2010). However, most of these methods are not readily extendable to non-linear chirps and in particular QFM chirps.

This paper discusses a model-dependent method each for parameter estimation of constant amplitude LFM and constant amplitude QFM chirps respectively. Subsequently, the results would show, separately, for a mixture of two LFM chirps and for a mixture of two QFM chirps, that even when correlation between $f(t)$ versus $t$ curves of two QFM chirps was as high as 0.999 , we could achieve accurate estimation of chirp-parameters which govern the individual $f(t)$. Estimation accuracy was within $\pm 1 \%$ for parameters of both the constituent chirps of the mixture.

The first method employs the Fractional Fourier Transform (FrFT) which was first proposed by Namias (1980). Applications of the FrFT in areas of signal processing have been covered by Almeida (1994). Ozaktas et al (1996) developed methods for efficient discrete computation of FrFT, similar to the Discrete Fourier Transform (DFT). The method proposed here for constant amplitude LFM chirps builds upon the work of Ozaktas et al (1996).

The second method, which is for estimating IF of individual QFM chirps (within a noisy mixture of QFM chirps) exploits the Generalized Time-Frequency Transform (GTFT) proposed by the authors of this paper (Shishir Sahay et al 2012a). 

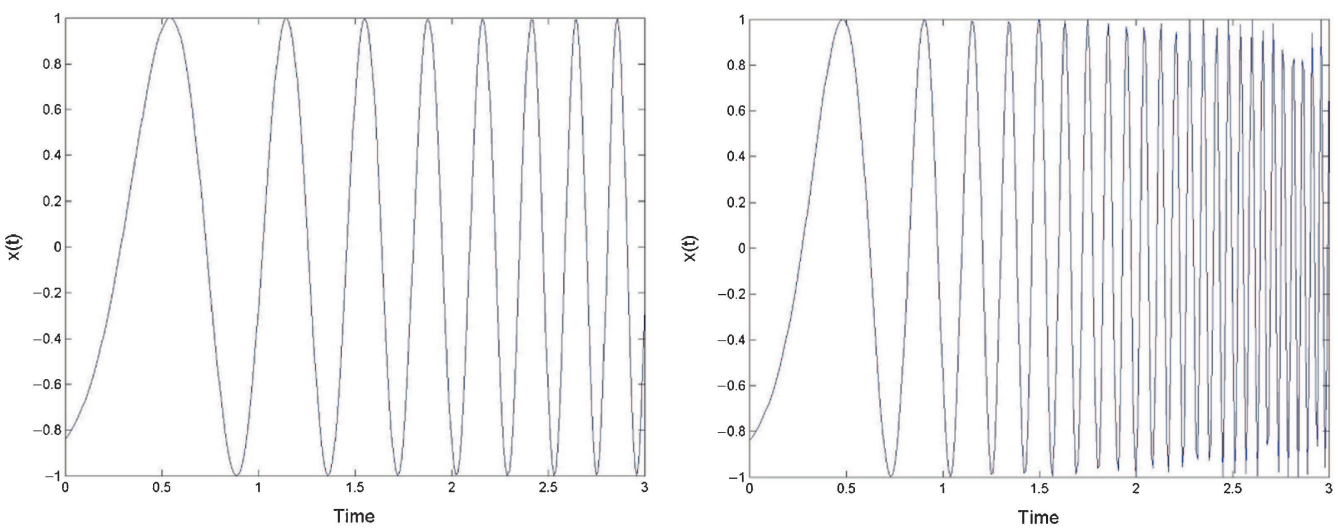

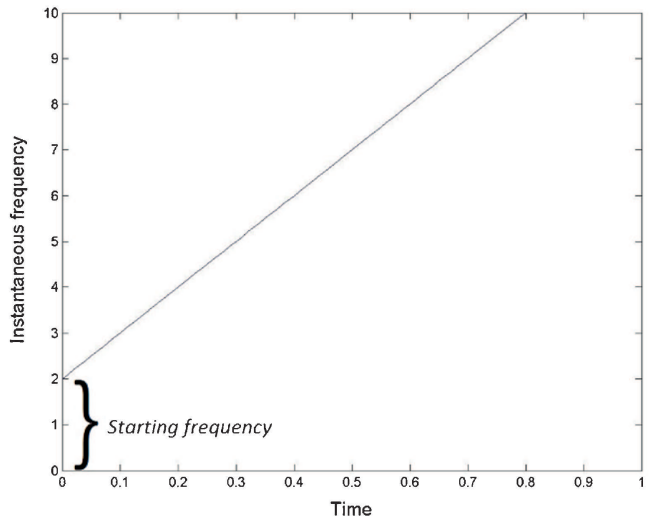

(a) LFM sinusoidal signal



(b) QFM sinusoidal signal

Figure 1. Time frequency representation of an LFM and QFM sinusoidal signal.

Section 2 introduces the FrFT from two perspectives - Almeida (1994) and Ozaktas et al (1996), whereas Section 4 introduces the GTFT. Sections 3 and 5 explain methods of IF estimation for a mixture of LFM chirps and for a mixture of QFM chirps respectively.

The problem at hand is explained with the aid of the following equation:

$$
y(t)=\sum_{i=1}^{M} A_{i} c_{i}(t) ; \quad \text { and received signal } \quad z(t)=y(t)+n(t),
$$

where

$$
\begin{aligned}
& y(t) \equiv \text { Mixture of LFM chirps (or mixture of QFM chirps) } \\
& \text { of different constant amplitudes. } \\
& M \equiv \text { Number of LFM chirps (or of QFM chirps) in the mixture. } \\
& A_{i} \equiv \text { Constant (w.r.t. time) amplitudes of chirps; } \\
& A_{1} \text { is strongest; } A_{2} \text { is next stronger and so on. } \\
& \text { i.e. } A_{1} \geq A_{2} \ldots \ldots \text {. } \\
& c_{i}(t) \equiv \text { LFM (or QFM) chirps. } \\
& z(t) \equiv \text { Received noisy mixture of chirps. } \\
& n(t) \equiv \text { Additive White Gaussian Noise. }
\end{aligned}
$$


The aim of the algorithm is to estimate $f(t)$ of the various $c_{i}(t)$ from the received $z(t)$.

The organization of the paper and steps involved in IF estimation of individual chirps (on receipt of a noisy mixture of chirps) are as follows.

1. Estimation of the bandwidth of $y(t)$ : Center frequency and One-sided bandwidth $B$ of $y(t)$ are estimated from frequency spectrum of $z(t)$. The center frequency and bandwidth are defined using the smallest band containing $98 \%$ of the total energy present in the frequency spectrum of $z(t)$. Estimate of bandwidth, $\hat{B}$ is employed during band-pass filtering to reject out-of-band noise. Pass-band of the filter is 1.1 times that of $\hat{B}$. Moreover, as shown in Eq. (23), $\hat{B}$ is utilized to restrict the search volume of unknown parameters.

2. Band-pass filtering of $z(t): z(t)$ is band-pass filtered to give $z_{F}(t)$ - filtered $z(t) \cdot z_{F}(t)=$ $y(t)+n_{F}(t)$, where $n_{F}(t)$ is the filtered noise.

3. Estimation of IF of stronger chirp: FrFT (for a mixture of LFM chirps) or GTFT (for a mixture of QFM chirps) of $z_{F}(t)$ is evaluated. From location of the strongest peak of the hypersurface over the parameter search region ( $\alpha$ and $u$ in case of LFM chirps; $\psi, \phi$ and $u$ in case of QFM chirps), IF estimation of the strongest chirp $c_{1}(t)$ is achieved. IF estimation is explained at Sections 3 and 5 for a mixture of LFM chirps and for a mixture of QFM chirps respectively. If the chirps are equally strong, any of them can be considered as the stronger chirp.

4. Separation of stronger chirp from $z_{F}(t)$ : Using the estimated parameters of $c_{1}(t)$, its synthetic version $c_{1_{S}}(t)$ is generated. Thereafter, amplitude $A_{1}$ of $c_{1}(t)$ is estimated. $c_{1_{S}}(t)$, weighted by the estimated amplitude $\hat{A}_{1}$ is separated from $z_{F}(t)$ at the correct time lag. Section 6 explains this method for the separation of chirps in a mixture of chirps which are closely located in the time-frequency plane.

5. IF estimation of weaker chirps and their successive removal: IF estimation and the separation for next stronger chirp is performed as explained in Steps 3 and 4. The procedures of parameter-estimation and separation are successively applied from the stronger chirps to the weaker ones. At each stage of evaluation of FrFT (or GTFT), maxima of the hyper-surface gives location of the parameters of the strongest chirp in the residual mixture of chirps and $n_{F}(t)$.

Section 7 presents the simulation results and Section 9 concludes the paper.

\section{Introduction to the Fractional Fourier Transform}

This Section gives an introduction to the FrFT from two similar perspectives - Almeida (1994) and Ozaktas et al (1996).

\subsection{The Fractional Fourier Transform - Almeida (1994)}

Almeida (1994) has defined the Fractional Fourier Transform (FrFT) by means of the transformation kernel given in the following equation:

$$
K_{\alpha}(t, u)= \begin{cases}\sqrt{\frac{1-j \cot (\alpha)}{2 \pi}} e^{j \frac{t^{2}+u^{2}}{2} \cot (\alpha)-j u t \csc (\alpha)} & \text { if } \alpha \text { is not a multiple of } \pi \\ \delta(t-u) & \text { if } \alpha \text { is a multiple of } 2 \pi ; \\ \delta(t+u) & \text { if }(\alpha+\pi) \text { is a multiple of } 2 \pi\end{cases}
$$


The FrFT for a general complex signal $x(t)$ is denoted by $X_{\alpha}(u)$ and its expression is shown in the equation

$$
X_{\alpha}(u)=\int_{-\infty}^{+\infty} x(t) \cdot K_{\alpha}(t, u) \mathrm{d} t
$$

Upon incorporating the three conditions for $\alpha$ of Eq. (4), into Eq. (5), we get the following equation:

$X_{\alpha}(u)= \begin{cases}\sqrt{\frac{1-j \cot (\alpha)}{2 \pi}} \cdot e^{j \frac{\cot (\alpha)}{2} u^{2}} \int_{-\infty}^{+\infty} x(t) e^{j \frac{\cot (\alpha)}{2} t^{2}} e^{-j u \cdot \csc (\alpha) \cdot t} \mathrm{~d} t & \text { if } \alpha \text { is not a multiple of } \pi ; \\ x(t) & \text { if } \alpha \text { is a multiple of } 2 \pi ; \\ x(-t) & \text { if }(\alpha+\pi) \text { is a multiple of } 2 \pi .\end{cases}$

2.1a Observations on Eqs. (4-6): From Eq. (4-6), we observe that:

1. Different values of $\alpha$ and $u$ result in different kernel functions.

2. FrFT $X_{\alpha}(u)$ of a signal $x(t)$ is a complex-valued three-dimensional surface over a plane dependent on two independent variables $\alpha$ and $u$ compared to the ordinary Fourier Transform $X(f)$ of a signal $x(t)$ which is a complex-valued two-dimensional curve with frequency $f$ as the independent variable.

3. If $x(t)$ happens to be the complex conjugate of a particular kernel function for some $\alpha$ and $u$, then $\left|X_{\alpha}(u)\right|$ would have a marked peak at that value of $\alpha$ and $u$. This comes from the fact that Eq. (5) has a form similar to Eq. (7), the dot product $\langle v(t), w(t)\rangle$ of two general complex signals $v(t)$ and $w(t)$.

$$
\langle v(t), w(t)\rangle=\int_{-\infty}^{+\infty} v(t) \cdot w^{*}(t) \mathrm{d} t, \quad \text { where } * \text { indicates conjugate. }
$$

4. From Eq. (6), we observe that when $\alpha=\pi / 2$, we obtain the equation of the ordinary Fourier Transform. We can thus consider the ordinary Fourier Transform operator as a change in representation of the signal corresponding to a counter-clockwise rotation of the time axis by $\pi / 2$. It is also noticed from Eq. (6) that when $\alpha=2 \pi$ (four times $\pi / 2$ ), we get the original signal $x(t)$. Other values of $\alpha$, thus imply a partial or fractional (fraction of $\pi / 2$ ) rotation of the time axis or fractionally applying the Fourier transform. Hence, the terminology "Fractional" Fourier Transform.

2.1b Discussion on properties of the Fractional Fourier Transform: Some properties of FrFT and kernel functions utilized in this text are as follows:

1. The kernel functions from Eq. (4) can be re-written as in the following equation:

$$
K_{\alpha}(t, u)=\sqrt{\frac{1-j \cot (\alpha)}{2 \pi}} e^{j\left[\frac{\cot (\alpha)}{2} t^{2}-u \cdot \csc (\alpha) \cdot t+\frac{u^{2}}{2} \cot (\alpha)\right]} .
$$

Thus, they form a family of LFM chirps of the general expression given by the following equation:

$$
e^{j\left(a t^{2}+b t+\theta\right)} .
$$


2. The kernel functions $K_{\alpha}(t, u)$ and $K_{\alpha}\left(t, u^{\prime}\right)$, taken as functions of t, for the same $\alpha$ but different values of the parameter $u$, form an ortho-normal set. This important property is shown in the following equation:

$$
\int_{-\infty}^{+\infty} K_{\alpha}(t, u) K_{\alpha}^{*}\left(t, u^{\prime}\right) \mathrm{d} t=\delta\left(u-u^{\prime}\right) .
$$

3. Assume that for a signal $x(t)$, we have the FrFT $X_{\alpha}(u)$, which, as we discussed, is a complexvalued three-dimensional surface. Consider the complex-valued curve $X_{\alpha_{1}}(u)$ corresponding to some $\alpha=\alpha_{1}$; it is a function in $u$. If we evaluate the FrFT of $X_{\alpha_{1}}(u)$ taking the kernel function to be $K_{-\alpha_{1}}(u, t)$, then we get $x(t)$. This is true for any value of $\alpha$. This is shown in Eq. (11). Note that the integration is on the variable $u$ similar to the integration on $f$ in the synthesis equation of the ordinary Fourier transform.

$$
x(t)=\int_{-\infty}^{+\infty} X_{\alpha}(u) K_{-\alpha}(u, t) \mathrm{d} u .
$$

From Eqs. (5-11), we see that $x(t)$ can be expressed in terms of the ortho-normal basis formed by LFM chirp signals $K_{\alpha}(t, u)$.

2.1c Application of the Fractional Fourier Transform to estimation of IF of LFM chirps: An important application of the FrFT is in estimation of the instantaneous frequency (IF) of linear frequency modulated (LFM) chirps. Merely evaluating the Fourier transform of a chirp signal, or for that matter any signal, does not convey the time-variation of IF. For this timefrequency representation, one normally uses a plane with two axes corresponding to time and frequency as shown in figure 1 for a LFM and a QFM sinusoidal signal. To understand the application of the FrFT to the estimation of LFM chirps, consider a signal $c(t)$, which is of the form given in Eq. (12). An example of such a chirp, $\sin \left(l t^{2}+m t+\lambda\right)$ is shown in figure 1(a).

$$
c(t)=e^{j\left(l t^{2}+m t+\lambda\right)} .
$$

Equation (13) shows the expression for IF $f(t)$ which is the derivative of the phase of the exponential in Eq. (12).

$$
f(t)=\frac{1}{2 \pi}(2 l t+m) \text { and } \omega\left(t_{\max }\right)=2 l t_{\max }+m
$$

where

$21 \equiv$ Chirp-rate of the LFM complex exponential.

$\mathrm{m} \equiv$ Starting-frequency $\omega\left(t_{0}\right)$ of the LFM complex exponential in $\mathrm{rad} / \mathrm{s}$ at $t=0$.

Assume that we receive $z(t)$, a noisy version of $c(t)$ and are interested in estimating Eq. (13) for $c(t)$. We first pass $z(t)$ through a suitable band-pass filter to reject out-of-band noise to give $z_{F}(t)$. We then evaluate the FrFT of $z_{F}(t)$ for different values of $\alpha$ and $u$. It is denoted as $Z_{F_{\alpha}}(u)$ and shown in the following equation:

$$
Z_{F_{\alpha}}(u)=\int_{-\infty}^{+\infty} z_{F}(t) K_{\alpha}(t, u) \mathrm{d} t .
$$


In light of Eqs. (5-9), we are in effect, evaluating the correlation of $z_{F}(t)$ with a family of LFM complex exponentials. Thus we have to perform a two parameter search to ascertain $\alpha$ and $u$ corresponding to the $K_{\alpha}(t, u)$, which has the best correlation with $z_{F}(t)$ and in turn to $c(t)$ (assuming noise is within limits). This happens, as explained, when $z_{F}(t)$ [and $c(t)$ to an approximate extent] and $K_{\alpha}(t, u)$ are complex conjugates of each other, resulting in a marked peak for $\left|Z_{F_{\alpha}}(u)\right|$ surface. Upon enforcing this conjugate requirement and comparing Eq. (12) with Eq. (8), we get the following equation:

$$
\begin{aligned}
& l=-\frac{\cot (\alpha)}{2} \\
& m=u \csc (\alpha) .
\end{aligned}
$$

Thus, from the values of $\alpha$ and $u$, say $\alpha_{1}$ and $u_{1}$ corresponding to maxima of $\left|Z_{F_{\alpha}}(u)\right|$ surface, we are able to ascertain $l$ and $m$ of the signal $c(t)$ from $z_{F}(t)$. Consequently, from Eq. (13), the starting-frequency $m$ is $u \csc (\alpha)$ and the chirp-rate $2 l$ is $-\cot (\alpha)$. It is to be noted that for an exponential LFM under investigation, there would be another maxima on the $\left|Z_{F_{\alpha}}(u)\right|$ surface over point, at $\alpha_{2}$ and $u_{2}$. Relationship between co-ordinates of the two maxima points is given by the following equation:

$$
\alpha_{1}=\alpha_{2}+\pi \text { and } u_{1}=-u_{2} \text {. }
$$

Either of the two maxima points $\left(\alpha_{1}, u_{1}\right)$ or $\left(\alpha_{2}, u_{2}\right)$ can be considered to ascertain $l$ and $m$ of the unknown chirp on account of the trigonometric identities in the following equation:

$$
\cot (\theta+\pi)=\cot (\theta) \text { and } \csc (\theta+\pi)=-\csc (\theta) .
$$

Since we are considering only the magnitude part of complex-valued FrFT surface i.e. $\left|Z_{F_{\alpha}}(u)\right|$, we have not equated $\lambda$ in Eq. (12) to the corresponding components of Eq. (8). The exhaustive two-parameter search to ascertain $\alpha$ and $u$ should be at resolution fine enough (of $u$ and $\alpha$ ) such that maxima of $\left|Z_{F_{\alpha}}(u)\right|$ surface is not missed. In practice, since $c(t)$ has limited support over time, as against $(-\infty,+\infty)$ demanded by Eq. (5), errors occur because of the limited time of integration. Similarly, results are erroneous if time-sampling is not sufficiently high during implementation of Eq. (5). Additionally, care is to be exercised for values of $\alpha$, which are integral multiples of $\pi$; Eq. (6) is to be used. Employing Eq. (5) for those values of $\alpha$ might seem misleading because $\cot (\alpha)$ in the kernel function given by Eq. (4) approaches $\pm \infty$ for those values of $\alpha$.

\subsection{Discrete implementation of the Fractional Fourier Transform - Ozaktas et al (1996)}

Ozaktas et al (1996) have discussed the methodology for the calculation of FrFT of a signal and have in fact proposed a discrete version of the FrFT (DFrFT), similar to the Discrete Fourier Transform (DFT) for the ordinary Fourier Transform. The variables and notation have been slightly modified compared to Almeida (1994). The $a^{\text {th }}$ order FrFT $\mathcal{F}^{a}[f(x)]$ of the function $f(x)$ for $0<|a|<2$ is given by the following equation:

$$
\mathcal{F}^{a}\left[f\left(x^{\prime}\right)\right] \equiv\left\{\mathcal{F}^{a} f\right\}(x) \equiv \int_{-\infty}^{+\infty} f\left(x^{\prime}\right) B_{a}\left(x, x^{\prime}\right) \mathrm{d} x^{\prime},
$$

where

$$
\begin{gathered}
B_{a}\left(x, x^{\prime}\right)=A_{\phi} e^{\left[j \pi\left(x^{2} \cot (\phi)-2 x x^{\prime} \csc (\phi)+x^{\prime 2} \cot (\phi)\right]\right.} \\
A_{\phi}=\frac{e^{(-j \pi \cdot \operatorname{sgn}(\sin (\phi)) / 4+j \phi / 2}}{|\sin (\phi)|^{1 / 2}} \text { and } \phi \equiv \frac{a \pi}{2} .
\end{gathered}
$$


Note that the function $f(x)$, whose FrFT has to be evaluated, has been written as $f\left(x^{\prime}\right)$ on the RHS of Eq. (19). Comparing Eq. (19) with Eq. (5), we get the equivalence of the two set of variables as shown in the following equation:

$$
x^{\prime} \equiv t, \quad x \equiv u, \quad \phi \equiv \alpha .
$$

For $a=1$, i.e. $\phi=\pi / 2$, the FrFT reduces to the ordinary Fourier Transform of $f(x)$ as shown in the following equation:

$$
\left\{\mathcal{F}^{a} f\right\}(x)=\int_{-\infty}^{+\infty} f\left(x^{\prime}\right) e^{\left(-j 2 \pi x x^{\prime}\right)} \mathrm{d} x^{\prime} .
$$

Similar to the earlier expression for FrFT by Almeida (1994), the FrFT is a complex-valued three-dimensional surface over a plane with axes $x$ and $a$. When estimating noisy LFM chirps using equations by Ozaktas et al (1996), the search for a maxima of the magnitude of complexvalued FrFT surface boils down to finding the corresponding $x$ and $a$.

Two efficient methods proposed by Ozaktas et al (1996) for discrete computation build upon the observation of Almeida (1994), that the expression in Eq. (6) corresponds to the following steps (for angles that are not multiples of $\pi$ ):

1. Multiplication of $x(t)$ by a LFM exponential signal $e^{j \frac{\cot (\alpha)}{2} t^{2}}$.

2. Fourier transform of the above function, with its argument scaled by $\csc (\alpha)$, to yield a function in $u$.

3. Product of this function with another LFM exponential signal $e^{j \frac{\cot (\alpha)}{2} u^{2}}$, the independent variable for which is $u$.

4. Product with a complex-valued amplitude factor.

Since the first method involves evaluation of Fresnel integrals, it has not been recommended by the authors themselves from the point of view of speed (Ozaktas et al 1996). Important steps in the second method are:

1. The time signal is shifted in time and centred at time $t=0$. Thereafter, appropriate scaling of the time signal ensures that the support of the signal in time domain and frequency domain is nearly the same. The sampling is such that the number of samples in both time and frequency domains is same, $\mathrm{N}$.

2. Appropriate sampling of the time signal is performed so that the Nyquist criterion is not violated for any of the intervening operations or signals.

3. Computationally efficient FFT algorithm is employed wherever feasible.

4. Finally, appropriate re-sampling is performed to cater for the effects of the initial procedures.

The work of Ozaktas et al (1996) can be studied for further details.

\section{Proposed method for estimation of parameters of single or proximally located LFM chirp signals}

During implementation, when the discrete method by Ozaktas et al (1996) is applied to estimation of instantaneous frequency (IF) of LFM chirps, the search, however and naturally so, still remains a two-parameter search for the appropriate $x$ and $a$ of Eq. (19). 
However if the chirps are proximally located on the time frequency plane, we propose to replace the two-parameter search by a simpler single-parameter search carried out in two steps. Henceforth, reference shall be made to the variables $u, \alpha$ and $t$ used by Almeida (1994) and not $x^{\prime}, x, a$ and $\phi$ used by Ozaktas et al (1996), unless otherwise specified. Equation (20) can always be referred to know the equivalence between the two sets of variables.

In the proposed method, an approximate value of $\alpha$ is first estimated separately, followed by an estimation of $u$ to ascertain the starting-frequency and chirp-rate of $c(t)$. Since, we have estimated an approximate value of $\alpha$, it is now not required to evaluate the FrFT over all values of $\alpha$ ranging from $-\pi$ to $+\pi$, thus resulting in considerable saving of computational time.

During the estimation of an LFM complex exponential of the form given by Eq. (12), it is seen from Eq. (15), the optimum $\alpha$, i.e. that value of $\alpha$ where a peak is expected for the $\left|Z_{F_{\alpha}}(u)\right|$ surface, would be given in the following equation:

$$
\alpha_{o p t}=\tan ^{-1}\left(\frac{-1}{2 l}\right) \pm n \pi, \text { where } n \text { is an integer. }
$$

However, for a LFM chirp of time duration T, the chirp-rate $2 l \approx \pm \hat{B} / T$, where $\hat{B}$ is the approximate one-sided bandwidth of the signal. The chirp-rate is positive if the IF is increasing with time; and negative otherwise. Hence, Eq. (23) is used to estimate the optimal value of $\alpha$.

$$
\alpha_{e s t}=\tan ^{-1}\left(\mp \frac{T}{\hat{B}}\right) \pm n \pi, \text { where } n \text { is an integer. }
$$

In the proposed modification to the normal procedure of evaluating $\left|Z_{F_{\alpha}}(u)\right|$ over all values of $\alpha$, an approximate value of $\alpha_{\text {opt }}$ is calculated from Eq. (23). Both the values of $\alpha_{\text {est }}$ are considered since there is no a priori information whether the chirp-rate of the unknown LFM is positive or negative. $\left|Z_{F_{\alpha}}(u)\right|$ is evaluated over a small interval of 0.1 radian on either side of the two values of $\alpha$.

Alternatively, a binary search procedure can be performed within both the 0.2 radian intervals. During this binary search, for each candidate-value $\alpha_{e s t}^{k}$ in the interval, we get a complex-valued curve $Z_{F_{\alpha_{e s t}^{k}}}(u)$ with $u$ as the independent variable. We consider only the magnitude part of the complex-valued curve, $\left|Z_{F_{\alpha_{e s t}^{k}}}(u)\right|$ and tabulate $\alpha_{e s t}^{k}$, along with the curve's maxima and corresponding value of $u$. We pick up the curve with the largest maxima amongst these set of curves. The corresponding values of $u$ and $\alpha$ are the ones we are interested in estimating. In other words, during the binary search, we are taking slices of the $\left|Z_{F_{\alpha}}(u)\right|$ surface along various values of $\alpha$ in the vicinity of the approximate values of $\alpha_{o p t}$ obtained from Eq. (23). All these slices result in curves with $u$ as the independent variable. We simply pick the curve with the largest maxima. For all the above calculations of $\left|Z_{F_{\alpha}}(u)\right|$, the second method of Ozaktas et al (1996) is employed. The values of $\alpha$ and $u$ so obtained are used to ascertain the chirp-rate $2 l$ and starting-frequency $m$ from Eq. (15) and Eq. (16) respectively.

Generally, in practical cases, the received noisy signal $z(t)$ is not of the form given by Eq. (12) but of the form $\cos \left(l t^{2}+m t+\lambda\right)$ or $\sin \left(l t^{2}+m t+\lambda\right)$. The relation given by Eq. (24) is used in such cases.

$$
\cos \left(l t^{2}+m t+\lambda\right)=\frac{1}{2}\left[e^{j\left(l t^{2}+m t+\lambda\right)}+e^{-j\left(l t^{2}+m t+\lambda\right)}\right]
$$

Variation of IF for the two LFM complex exponentials on RHS of Eq. (24) is shown in figure 2 (for $l, m>0$ ). 
We have discussed at Eqs. (17 and 18) that an exponential LFM results in two maxima for the $\left|Z_{F_{\alpha}}(u)\right|$ surface. Similarly, in light of Eq. (24), when we take FrFT of LFM sinusoidal signals $\cos \left(l t^{2}+m t+\lambda\right)$ or $\sin \left(l t^{2}+m t+\lambda\right)$, we get four maxima on the $\left|Z_{F_{\alpha}}(u)\right|$ surface. However, from Eq. (24), on account of the 1/2 multiplying factor, the value of maxima would be lesser by a factor of 2 . This situation is similar to the case for ordinary sinusoidal signals $\cos (2 \pi f t+\lambda)$ and $\sin (2 \pi f t+\lambda)$. For them, we obtain two impulses in the ordinary Fourier transform complex-valued curve at frequencies $-f$ and $+f$, whereas there is only one impulse at $+f$ for the complex exponential signal of frequency $f$.

During implementation, for the discrete evaluation of FrFT for a set or range of values of $\alpha$, the second method proposed by Ozaktas et al (1996) was employed. The computer code of the related function was sourced from Ozaktas code. Upon implementation of the code, one slice of the three-dimensional FrFT surface is generated for all values of $u$ for the supplied value of $a$ which is related to $\alpha$ by $\alpha=a \pi / 2$ (refer Eqs. 19 and 20). Values of $\alpha$ in vicinity of $\alpha_{\text {est }}$ are employed for generation of these various slices (curves with $u$ as the independent variable). The two-dimensional curve with the highest maxima is selected and the center-frequency of the LFM chirp is calculated from Eq. (25). Since the time of duration of the LFM chirp and its chirp-rate are known, the starting frequency can be easily estimated from the center frequency (figure 2).

$$
l\left(T_{1}+T_{2}\right)+m=\frac{\frac{N}{2}-n_{0}}{T} \csc \left(-\alpha \cdot \frac{\pi}{2}\right),
$$

where $N$ is the number of samples, $T=T_{2}-T_{1}$ is the time-duration of the sampled LFM chirp of the form $\sin \left(l t^{2}+b t+\lambda\right)$ and $n_{0}$ is the location of the peak of the two-dimensional curve having the highest value of maxima, i.e. the curve $\left|Z_{F_{\alpha_{e s t} t_{\text {opt }}}}(u)\right|$.

\section{Introduction to Generalized Time-Frequency Transform (GTFT)}

Shishir Sahay et al (2012a) have proposed a generalized time-frequency transform inspired by the FrFT. The kernels of several transforms such as the Fresnel Transform, Gauss-Weierstrass Transform and Special Affine Fourier Transform (Cai 2000), were also compared to the FrFT kernel. To encompass all these transforms, a new class of transforms was proposed, whose general kernel is given by the following equation:

$$
K_{\phi}(x, y)=k_{0}(\phi) e^{j\left[h_{1}(y, \phi)-k_{1}(\phi) x y+h_{2}(x, \phi)\right]},
$$

where $h_{1}, h_{2}$ are real-valued functions which depend on the real parameter $\phi . k_{0}, k_{1}$ are dependent on the real parameter $\phi$ and are constants with respect to $x$ and $y$.

The kernel of the FrFT can be obtained as a special case of Eq. (26) by selecting the values as in the following equation:

$$
\begin{aligned}
& k_{0}(\phi)=\sqrt{\frac{1-j \cot (\phi)}{2 \pi}} \text { and } k_{1}(\phi)=\csc (\phi) \\
& h_{1}(z, \phi)=h_{2}(z, \phi)=\frac{z^{2}}{2} \cot (\phi) .
\end{aligned}
$$

The kernels of several other transforms such as the Fresnel Transform and Special Affine Fourier Transform (Cai 2000) are also realizations of Eq. (26).

The kernel of the GTFT is given in the following equation:

$$
K_{\phi, \psi}(t, u)=\sqrt{\frac{1}{2 \pi j l(\phi)}} e^{j\left[u^{2} g(\phi)+t^{2} g(\phi)-u t \frac{1}{l(\phi)}+f(u, \psi)-f(t, \psi)\right]} .
$$





Figure 2. Variation of IF for $e^{j\left(l t^{2}+m t+\lambda\right)}$ and $e^{-j\left(l t^{2}+m t+\lambda\right)}$, for $l, m>0$.

The GTFT satisfies the properties of invertibility and index-additivity similar to the FrFT. For a particular value of $u$, the kernel is a function of time, parametrized by $\phi$ and $\psi$. Moreover, comparing Eq. (28) to Eq. (26), we gain an additional degree of freedom by parametrizing $f(\cdot)$ and getting a family of transforms. In the estimation of IF of an incoming unknown chirp signal of known model, we can use this very extra freedom to handle non-linearities in the nature of instantaneous frequency. Thus, we are not limited, to estimating instantaneous frequency of unknown chirps with linear frequency modulation, as in the case of FrFT.

We already know that for FrFT, if $X_{\alpha}(u)$ is the FrFT of $x(t)$ at angle $\alpha$, then, the FrFT of $x^{1}(t)=x\left(t-t_{0}\right)$ is

$$
X_{\alpha}^{1}(u)=X_{\alpha}\left(u-t_{0} \cos \alpha\right) e^{j \frac{t_{0}^{2}}{2} \sin (\alpha) \cos (\alpha)-j u t \sin (\alpha)} .
$$


We try to find a similar property for the GTFT corresponding to a quadratic chirp.

Suppose $Y_{\alpha}(u)$ is the GTFT of $y(t)$ at an angle $\alpha$ with $f(t)=k t^{3}$, for a constant $k$, then the GTFT of $y^{1}(t)=y\left(t-t_{0}\right)$ at the angle $\alpha$ is

$$
Y_{\alpha}^{1}(u)=Y_{\beta}(\mu) e^{j g\left(u, k, t_{0}, \alpha\right)},
$$

where $\beta, \mu$ and $g\left(u, k, t_{0}, \alpha\right)$ are defined as

$$
\begin{aligned}
& \cot \beta=\cot \alpha-6 k t_{0} \\
& \mu=\sin \beta\left(u \csc \alpha+3 k t_{0}^{2}-t_{0} \cot \alpha\right) \\
& g\left(u, k, t_{0}, \alpha\right)=\frac{u^{2}}{2} \cot \alpha+k u^{3}-u t_{0} \csc \alpha+\frac{t_{0}^{2}}{2} \cot \alpha-k t_{0}^{3}-\frac{\mu^{2}}{2} \cot \beta-k \mu^{3} .
\end{aligned}
$$

Practically, we may not always evaluate the transform in a time synchronised manner. In such cases, this property is extremely important to visualize the transform. The proof of this property is included in Appendix A.1.

\subsection{Eigenfunctions of the GTFT}

Kernel of the GTFT is given by

$$
\begin{aligned}
K_{\phi, f}(t, u) & =\sqrt{\frac{1-j \cot \phi}{2 \pi}} e^{j\left[\frac{u^{2}}{2} \cot (\phi)+\frac{t^{2}}{2} \cot (\phi)-u t \csc (\phi)+f(u)-f(t)\right]} \\
& =K_{\phi}(t, u) e^{j(f(u)-f(t))} .
\end{aligned}
$$

where $K_{\phi}(t, u)$ is kernel of the FRFT.

A possible choice for the eigenfunctions of the Fractional Fourier Transform operator $\mathcal{F}$ that is generally agreed upon is given by the set of normalized Hermite-Gauss functions (Bultheel \& Martinez 2003):

where

$$
\Phi_{n}(x)=\frac{2^{1 / 4}}{\sqrt{2^{n} n !}} e^{-x^{2} / 2} H_{n}(x),
$$

$$
H_{n}(x)=(-1)^{n} e^{x^{2}} \mathcal{D}^{n} e^{-x^{2}}, \quad \mathcal{D}=\frac{\mathrm{d}}{\mathrm{d} x}
$$

is a Hermite polynomial of degree $n$. The corresponding eigenvalues for FrFT at angle $\alpha=a \pi / 2$ are

$$
\lambda_{a, n}=e^{-i n a \pi / 2}=\lambda_{a}^{n}=\lambda_{n}^{a} .
$$

The set of eigenfunctions of the FrFT form an orthonormal basis for the functions in $\mathcal{L}$. This allows us to form an alternate representation of kernel of the FrFT given by

$$
K_{a}(u, t)=\sum_{n=0}^{+\infty} \lambda_{n}^{a} \Phi_{n}(u) \Phi_{n}(t) .
$$

For the GTFT, consider the set of functions given by

$$
\Psi_{n}(x)=\Phi_{n}(x) e^{j f(x)},
$$

where $\Phi_{n}(x)$ and $f(x)$ are as defined earlier.

We find that these functions are the eigenfunctions of the GTFT.

$$
\text { i.e. } \int_{-\infty}^{\infty} \Psi_{n}(t) K_{\alpha, f}(u, t) \mathrm{d} t=\sigma_{a, n} \Psi_{n}(u) .
$$


And, the eigenvalues are given by

$$
\sigma_{a, n}=\lambda_{a, n}=e^{-i n a \pi / 2}
$$

Proof:

$$
\begin{aligned}
\text { i.e. } \int_{-\infty}^{\infty} \Psi_{n}(t) K_{\alpha, f}(u, t) \mathrm{d} t=\int_{-\infty}^{\infty} & \Phi_{n}(t) e^{j f(t)} * K_{\alpha}(u, t) e^{j(f(u)-f(t))} \mathrm{d} t \\
= & \int_{-\infty}^{\infty} \Phi_{n}(t) K_{\alpha}(u, t) e^{j f(u)} \mathrm{d} t \\
= & e^{j f(u)} \int_{-\infty}^{\infty} \Phi_{n}(t) K_{\alpha}(u, t) \mathrm{d} t \\
& =e^{j f(u)} \Phi_{n}(u) \lambda_{a, n} \\
& =\Psi_{n}(u) \lambda_{a, n}
\end{aligned}
$$

\section{Proposed method for estimation of parameters of QFM chirp signals}

The quadratic nature of instantaneous frequency (IF), results in a chirp signal of the form given by the following equation:

$$
c(t)=e^{j\left(a t^{3}+b t^{2}+c t+\lambda\right)} .
$$

In the practical case, we have signals of the form given by the following equation:

$$
c(t)=\cos \left(a t^{3}+b t^{2}+c t+\lambda\right) \text { or } c(t)=\sin \left(a t^{3}+b t^{2}+c t+\lambda\right) .
$$

Variation of IF for the QFM case is given by the following equation:

$$
f(t)=\frac{1}{2 \pi}\left(3 a t^{2}+2 b t+c\right) \text { and } \omega\left(t_{\max }\right)=3 a\left(t_{\max }\right)^{2}+2 b\left(t_{\max }\right)+c .
$$

To use the GTFT for the estimation of QFM chirps, we will use the form of the kernel given in the following equation:

$$
K_{\phi, \psi}(t, u)=\sqrt{\frac{\csc (\phi)}{2 \pi j}} \cdot e^{j\left[u^{2} \cdot \frac{\cot (\phi)}{2}+t^{2} \cdot \frac{\cot (\phi)}{2}-u t \csc (\phi)+\psi u^{3}-\psi t^{3}\right]},
$$

where comparing Eq. (28) and Eq. (39),

$$
\begin{aligned}
l(\phi) & \equiv \sin (\phi) \\
f(u, \psi) & \equiv \psi u^{3} \\
f(t, \psi) & \equiv \psi t^{3} \\
g(\phi) & \equiv \frac{\cot (\phi)}{2}
\end{aligned}
$$

Similar to the treatment of LFM chirps, using the kernel given in Eq. (39), $Z_{F_{\phi, \psi}}(u)$ is evaluated in Eq. (40) to evaluate parameters of the individual $c(t)$. As mentioned earlier, it is known a priori that the chirp is a QFM chirp.

$$
Z_{F_{\phi, \psi}}(u)=\int_{-\infty}^{+\infty} z_{F}(t) \cdot K_{\phi, \psi}(t, u), \mathrm{d} t
$$

Substituting the kernel expression from Eq. (39) into Eq. (40), we get the following equation:

$$
Z_{F_{\phi, \psi}}(u)=\sqrt{\frac{\csc (\phi)}{2 \pi j}} \int_{-\infty}^{+\infty} z_{F}(t) \cdot e^{j\left[-\psi t^{3}+t^{2} \cdot \frac{\cot (\phi)}{2}-u t \csc (\phi)+u^{2} \cdot \frac{\cot (\phi)}{2}+\psi u^{3}\right]} \mathrm{d} t .
$$


The kernel of the GTFT optimized for QFM chirps at $\phi=0$ becomes a delta function, so the GTFT becomes an identity function, similar to the FrFT.

$Z_{F_{\phi, \psi}}(u)$ is a complex-valued four-dimensional hyper-surface compared to the threedimensional complex-valued surface obtained upon evaluating FrFT and the two-dimensional complex-valued curve obtained upon evaluating the ordinary Fourier transform of a signal. Similar to the discussion of estimation of noisy LFM signal using FrFT, we would get a maxima for the $\left|Z_{F_{\phi, \psi}}(u)\right|$ surface, when the kernel and $z_{F}(t)$ [and in turn $c(t)$, if noise is within limits] are complex conjugates of each other. Comparing Eq. (36) and Eq. (41), we get the following equations:

$$
\begin{gathered}
a=\psi \\
b=-\frac{\cot (\phi)}{2} \\
c=u \csc (\phi) .
\end{gathered}
$$

Therefore, as against a two-parameter search in case of LFM chirps, we need to implement a three-parameter search in case of QFM chirps.

\subsection{Practical implementation of GTFT}

Equation (41) can be rewritten as the following equation:

$$
Z_{F_{\phi, \psi}}(u)=\sqrt{\frac{\csc (\phi)}{2 \pi j}} \cdot e^{j \psi u^{3}} \int_{-\infty}^{+\infty}\left(z_{F}(t) \cdot e^{-j \psi t^{3}}\right) e^{j\left[t^{2} \frac{\cot (\phi)}{2}-u t \csc (\phi)+\frac{u^{2}}{2} \cot (\phi)\right]} \mathrm{d} t .
$$

In pursuit of the implementation of Eq. (45), the following operations are performed on the filtered noisy signal $z_{F}(t)$.

1. Multiplication of $z_{F}(t)$ with a chirp $e^{-j \psi t^{3}}$ - different candidate values of $\psi$ are considered.

2. FrFT is evaluated for $\left(z_{F}(t) \cdot e^{-j \psi t^{3}}\right)$ based on the efficient second method by Ozaktas et al (1996). For the correct value of $\psi$, indicated in Eq. (42), the QFM chirp would reduce to an LFM chirp. $\left|Z_{F_{\phi, \psi}}(u)\right|$ would therefore display a marked peak indicating that the unknown QFM chirp is conjugate of the kernel function $K_{\phi, \psi}(t, u)$ with the chosen parameters. Evaluation of the FrFT would result in a function in $u$.

3. Multiplication of the above function in $u$ with a quadratic chirp $e^{j \psi u^{3}}$.

4. Multiplication by a complex amplification factor.

As in the case of LFM chirps, where we do not evaluate FrFT of all values for $\alpha$ but only over a restricted range given by Eq. (23), here too we evaluate FrFT for a restricted range of $\phi$ given by Equation (46).

$$
\phi_{\text {est }} \approx \tan ^{-1}\left(\mp \frac{T}{\hat{B}}\right) \pm n \pi, \text { where } n \text { is an integer. }
$$

Note that $2 b \approx \hat{B} T$ in this case similar to the relation $2 l \approx \hat{B} T$ in the LFM case. However, the approximation is less accurate compared to the LFM case. Hence, the search region around the optimum value of $\phi$ is taken to be 0.4 radian and not 0.2 radian as in the LFM case.

For QFM chirps of the form given in Eq. (37), the same discussion holds as in the case of LFM chirps of the form given in Eq. (24). 
Additionally, the GTFT can also be used to estimate variation of IF for higher order chirps. This is achieved by exploiting the freedom available for the choice of function $f(\cdot)$ in Eq. (28). Efficient search for the optimal parameters of the kernel can be performed by using the technique of Particle Swarm Optimization and its various modifications.

\section{Separation of individual chirps from a mixture of chirps}

When we evaluate the FrFT (or GTFT) of the chirp mixture, the multiple chirps would be indicated by multiple maxima on the $\left|Z_{F_{\alpha}}(u)\right|$ (or $\left|Z_{F_{\phi, \psi}}(u)\right|$ ) surface. From the corresponding values of $\alpha$ and $u$ (or $\psi, \phi$ and $u$ ), parameters of the multiple chirps can be ascertained. However, when the multiple chirps are located in close proximity on the time-frequency plane then the multiple peaks are not clearly distinguishable on the transform surface. The situation is aggravated further in cases of poor SNR (composite chirp-mixture power to noise power) and more so when one chirp is far stronger in amplitude compared to the others. This strong chirp acts like a strong interferer for other chirps, degrading the SNR further for the weaker chirps.

Methods in literature comprise of either forcing to zero all values on the transform surface, which are in the vicinity of and including the stronger peak; or on the contrary, forcing to zero all values on the transform surface except those in the vicinity of and including the stronger peak (Roshen Jacob et al 2009). In the former case, subsequently evaluating the inverse transform results in detection of the weaker chirps provided that the SNR of the original noisy mixture was not very bad. Detection of the weaker chirp by this procedure is facilitated because a very strong interferer, the stronger chirp, has been weeded out. In the latter case, evaluating the inverse transform results in a time signal which comprises of only the stronger chirp. It is then removed from the mixture and the transform evaluated again for the residual mixture to estimate the weaker chirps.

Clearly, when the multiple chirps (weaker and stronger ones) are very close on the timefrequency plane, both the methods of chirp-separation fail. In the former case, the weaker chirps, co-located with the stronger chirp, are unknowingly weeded out. In the latter case, the weaker chirps, co-located with the stronger chirp, are also retained; hence when we evaluate the inverse transform, we still get a mixture of the stronger and the weaker chirp, albeit with an improved ratio of sum of powers of all chirps to that of noise.

A different method to separate the chirps was tried with encouraging results. As we had discussed in Section 1, $z(t)$ is passed through a band-pass filter to get $z_{F}(t)$. The following steps are involved in the proposed method of chirp-separation.

1. FrFT (or GTFT) of $z_{F}(t)$ is evaluated and parameters of the strongest chirp $c_{1}(t)$ are ascertained from location of the strongest peak of the $\left|Z_{F_{\alpha}}(u)\right|$ (or $\left|Z_{F_{\phi, \psi}}(u)\right|$ ) surface. $c_{1}(t)$ is then synthetically generated. This can easily be done, since all its parameters except the constant component of phase $\lambda$ is unknown - refer Eqs. (12 and 36). This synthetic chirp is denoted by $c_{1 s}(t)$, where subscript ' $s$ ' denotes 'synthetic' and ' 1 ' indicates 'chirp- 1 '.

2. Correlation of $c_{1_{S}}(t)$ is checked with $z_{F}(t)$. This is checked for various incremental timelags $\tau$ of $c_{1_{S}}(t)$ to cater for the unknown phase $\lambda$. Equation (47) is employed to ascertain correlation coefficient $\rho_{\left\langle C_{1_{S}}, Z_{F}\right\rangle}(\tau)$, which varies from -1 for two exactly "opposing" signals to +1 for two exactly "similar" signals.

$$
\rho_{\left\langle C_{1_{S}}, Z_{F}\right\rangle}(\tau)=\frac{1}{\sqrt{E_{c_{1_{S}}} \cdot E_{z_{F}}}} \int_{T} c_{1_{S}}(t-\tau) \cdot z_{F}^{*}(t) \mathrm{d} t,
$$


where * indicates conjugation, $T$ is the time duration of the two signals; and $E_{c_{1 S}}$ and $E_{z_{F}}$ are energies of the two signals $c_{1_{S}}(t)$ and $z_{F}(t)$.

Evaluation of $\rho_{\left\langle C_{1}, Z_{F}\right\rangle}(\tau)$ for all the values of $\tau$ can be easily achieved by using MATLABR.

3. Time-lag $\tau_{\rho_{\max }}$ corresponding to best correlation $\rho_{\left\langle C_{1_{S}}, Z_{F}\right\rangle}\left(\tau_{\rho_{\max }}\right)$ is ascertained from maxima of the curve $\rho_{\left\langle C_{1_{S}}, Z_{F}\right\rangle}(\tau)$ At this stage, we can safely say that $c_{1_{S}}(t) \approx c_{1}(t)$. Thereafter $c_{1_{S}}(t)$ is subtracted from $z_{F}(t)$ to give a residual mixture $z_{F_{R}}(t)$ comprising only of $n_{F}(t)$ and the weaker chirps $A_{i} c_{i}(t), i=2, .3 \ldots M$. However, a word of caution - knowledge of the strength (amplitude) $A_{1}$ of $c_{1}(t)$ is also important. Merely subtracting $c_{1_{S}}(t)$ without any amplification $A_{1}$ would not cancel presence of $c_{1}(t)$ in $z_{F}(t)$.

4. Estimation of unknown amplitude of stronger chirp. To evaluate the unknown amplitude $A_{1}$ of $c_{1_{S}}(t)$ to be employed for subtraction, $c_{1_{S}}(t)$ with unit amplification is generated and its transform (FrFT or GTFT) evaluated. Magnitude of peak of this transform surface is compared with peak of transform of $z_{F}(t)$. Ratio of latter magnitude to the former gives an estimate $\hat{A}_{1}$ of magnitude of $c_{1}(t)$ in the chirp-mixture $y(t)$. The chosen $\hat{A}_{1}$ of $c_{1_{S}}(t)$ needs to be reconfirmed. For this, Eq. (48) is used to evaluate the residual signal $z_{F_{R}}(t)$ after removal of $c_{1_{S}}(t)$ at the estimated amplification $\hat{A}_{1}$ of $c_{1}(t)$.

$$
z_{F_{R}}(t)=z_{F}(t)-\hat{A}_{1} c_{1_{S}}(t)
$$

The correlation coefficient of $z_{F_{R}}(t)$ with $c_{1_{S}}(t)$ at the earlier calculated time-lag $\tau_{\rho_{\max }}$ i.e. $\left.\rho_{\left\langle c_{1 S}\right.}, z_{F_{R}}\right\rangle\left(\tau_{\rho_{\max }}\right)$, should be as small as possible to be sure that complete removal of $c_{1_{S}}(t)$ and in turn of $c_{1}(t)$ at the proper amplification has occurred. To ensure the same, an iterative procedure is employed. Conceptually, the procedure involves removal from $z_{F}(t)$ of $c_{1_{S}}(t)$ at different candidate-values of amplification to give a residual mixture. Correlation of this residual mixture is ascertained with $c_{1_{S}}(t)$ at the earlier evaluated time-lag $\tau_{\rho_{\max }}$. That candidate-value of amplification is selected for which this correlation is the least. Figure 3 shows the correlation between $z_{F_{R}}(t)$ and $c_{1_{S}}(t)$ for different candidate-values of $\hat{A}_{1}$ from 0.25 to 20 in steps of 0.25 . Two LFM chirps were considered for five different values of $A_{2} / A_{1}$ ratios from $1 / 10$ to $9 / 10$ in steps of $2 / 10$. It can be seen that there is a marked minima for all the curves. A steepest descent algorithm can be applied to ascertain that value of $\hat{A}_{1}$ in Eq. 48 for which least correlation exists between $z_{F_{R}}(t)$ and $c_{1_{S}}(t)$. For each iteration step $k$ of the iterative procedure, we choose a value for $A_{1 s_{k}}$ (subscript ' 1 ' indicates 'chirp-1', 's' indicates 'synthetic' and ' $k$ ' indicates the ' $k$ th iteration number'). For the first iteration, $A_{1 s_{1}}$ is chosen to be $\hat{A}_{1}$, which was evaluated at the beginning of Step 4. At each $k^{\text {th }}$ step of the iterative procedure the following is performed.

(a) $c_{1_{S}}(t)$ after weighting by $A_{1 s_{k}}$ is subtracted at time-lag $\tau_{\rho_{\max }}$ from $z_{F}(t)$ to give a residual mixture $z_{F_{R_{k}}}(t)$ comprising of the filtered noise, weaker chirps and possibly some unknown portion $\gamma_{k}$ of $c_{1}(t)$. The various relationships are shown in the following equation:

$$
\begin{aligned}
z_{F_{R_{k}}}(t) & =z_{F}(t)-A_{1 s_{k}} c_{1_{S}}(t) \\
& =n_{F}(t)+\gamma_{k} c_{1}(t)+\sum_{i=2}^{M} A_{i} c_{i}(t), \\
\text { where } \gamma_{k} & =A_{1}-A_{1 s_{k}} .
\end{aligned}
$$




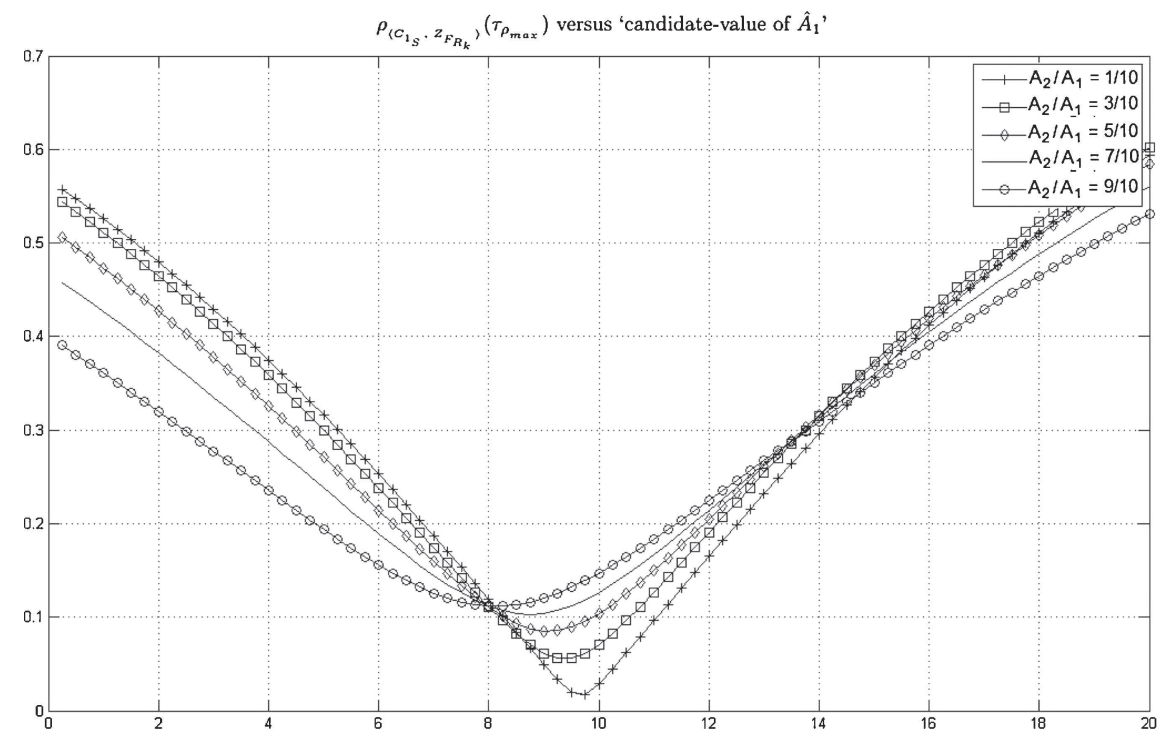

Figure 3. $\left.\left.\rho_{\left\langle C_{1},\right.}, Z_{F_{R_{k}}}\right\rangle, \tau_{\rho_{\max }}\right)$ for different $\hat{A}_{1}$ chosen for separation of stronger $c_{1}(t)$ from a mixture of two LFM chirps. Five different $A_{2} / A_{1}$ ratios have been considered.

(b) $\rho_{\left\langle C_{1}, Z_{F_{R_{k}}}\right\rangle}\left(\tau_{\rho_{\max }}\right)$, i.e. correlation coefficient at time-lag $\tau_{\rho_{\max }}$ (ascertained in Step 3) of $c_{1_{S}}(t)$ with $z_{F_{R_{k}}}(t)$, is checked. Equation (50) is to be employed and not

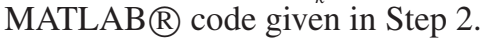

$$
\rho_{\left\langle C_{1_{S}}, Z_{F_{R_{k}}}\right\rangle}\left(\tau_{\rho_{\max }}\right)=\frac{1}{\sqrt{E_{c_{1_{S}}} \cdot E_{z_{F_{R_{k}}}}}} \int_{T} c_{1_{S}}\left(t-\tau_{\rho_{\max }}\right) \cdot z_{F_{R_{k}}}^{*}(t) \mathrm{d} t .
$$

(c) An example of the $\left.\left.\rho_{\left\langle C_{1},\right.}, Z_{F_{R_{k}}}\right\rangle, \tau_{\rho_{\max }}\right)$ versus 'candidate-value of $\hat{A}_{1}$ ' curve is shown in figure 3. Slope of the curve at $A_{1 s_{k}}$ is ascertained by evaluating Eq. (49) and Eq. (50) for a candidate-value very close to the presently chosen $A_{1 s_{k}}$. This slope is used, in accordance with the steepest descent algorithm, to choose the next value i.e. $A_{1 s_{(k+1)}}$. Nature of the slope (positive or negative) and its value determine the choice of $A_{1 s_{(k+1)}}$. If the chosen value of $A_{1 s_{k}}$ happens to the left of the minima in figure 3 (i.e. slope is negative at $A_{1 s_{k}}$ ), then the jump from $A_{1 s_{k}}$ to $A_{1 s_{(k+1)}}$ would be 'rightwards'; and 'leftwards' if the chosen value happens to be to the right of the minima. Moreover, higher the absolute value of magnitude of slope, higher the 'jump' to choose $A_{1 s_{(k+1)}}$. Relation between $A_{1 s_{k}}$ and $A_{1 s_{(k+1)}}$ is given in Eq. (51), where $\mu$ is a user-chosen 'jump parameter' and $g\left(A_{1 s_{k}}\right)$ is the slope of the $\rho_{\left\langle C_{1},\right.}, Z_{\left.F_{R_{k}}\right\rangle}\left(\tau_{\rho_{\max }}\right)$ versus 'candidatevalue of $\hat{A}_{1}$ ' curve at $\hat{A}_{1}=A_{1 s_{k}}$. Choosing a higher value of $\mu$ results in faster convergence to the minima point. However, if $\mu$ is excessively large, then there is a danger of oscillation of the successive values of $A_{1 s_{k}}$ around the minima. It is therefore advisable to choose a small value of $\mu$.

$$
A_{1 s_{(k+1)}}=A_{1 s_{k}}-\mu \cdot g\left(A_{1 s_{k}}\right), \text { where } \mu>0 .
$$


(d) Amplitude $A_{1 s_{k}}$ corresponding to that iteration $k$ is selected for which $A_{1 s_{k}} \approx A_{1 s_{(k-1)}}$. In this case $A_{1 s_{k}} \approx A_{1}$. This indicates that for the selected $A_{1 s_{k}}$ for $c_{1_{S}}(t)$, the corresponding $z_{F_{R_{k}}}(t)$ contains least component $\gamma_{k}$ of $c_{1}(t)$. From Eq. (49) $A_{1 s_{k}} \approx A_{1}, \Rightarrow$ $\gamma_{k} \approx 0$.

5. $c_{1_{S}}(t)$ with above-selected amplification $A_{1 s_{k}}$ and time-lag $\tau_{\rho_{\max }}$ is then subtracted from $z_{F}(t)$ to give $z_{F_{R}}(t)$ with negligible component of $c_{1}(t)$.

6. Transform of $z_{F_{R}}(t)$ is then evaluated. Peak corresponding to the next stronger $\operatorname{chirp} c_{2}(t)$ is then discernible on the transform surface.

The above method of chirp-separation is performed successively from the stronger to the weaker chirp. In every stage of chirp-separation, SNR for the weaker chirps in the residual is improving because a stronger interferer - the stronger chirp is getting "weeded out".

\section{Results}

\subsection{LFM chirps}

7.1a Identification of chirp parameters in a mixture of LFM chirps: Consider the case in which the signal is a mixture of three different linear chirps,

$$
y(t)=e^{2 \pi j\left(50 t^{2}-100 t\right)}+2 e^{2 \pi j\left(128 t^{2}+200 t\right)}+e^{2 \pi j\left(200 t^{2}+45 t\right)}
$$

$y(t)$ is sampled between $0 s$ and $2 s$ at sampling frequency of $1000 \mathrm{~Hz}$. DFrFT of samples of $y(t)$ is calculated for $\alpha$ in range $[-1,0]$ and surface obtained is shown in figure 4 .

In figure 4 we can see the peaks corresponding to the different linear chirps, but we also see many more peaks than expected, this is because of our low sampling frequency. The chirp characteristics can be calculated using the concepts of $\alpha_{\text {opt }}$ and centralfrequency $(C f)$. Figure 5 plots the maximum magnitude at an alpha vs alpha for low sampling frequency of $1000 \mathrm{~Hz}$.

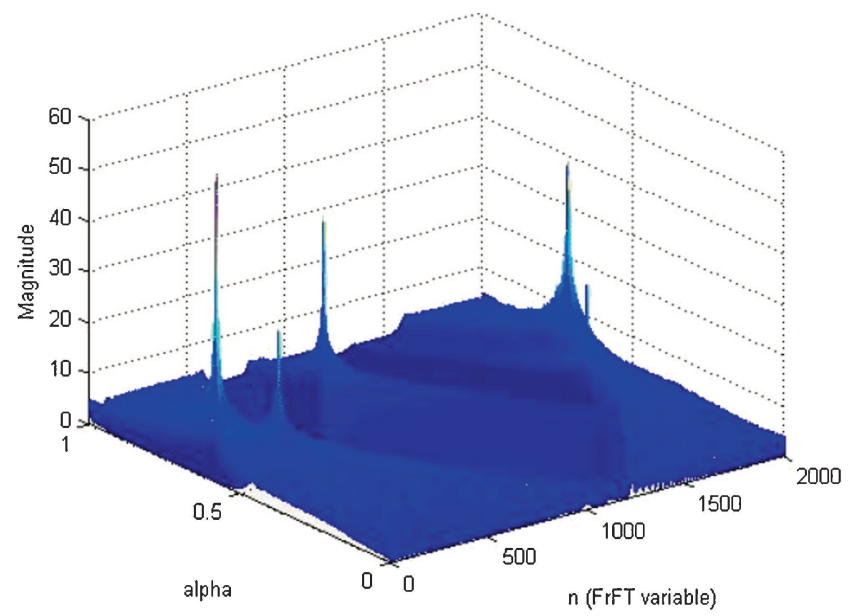

Figure 4. Estimation of chirp characteristics in a mixture of non-proximally located linear chirps(low sampling frequency). 


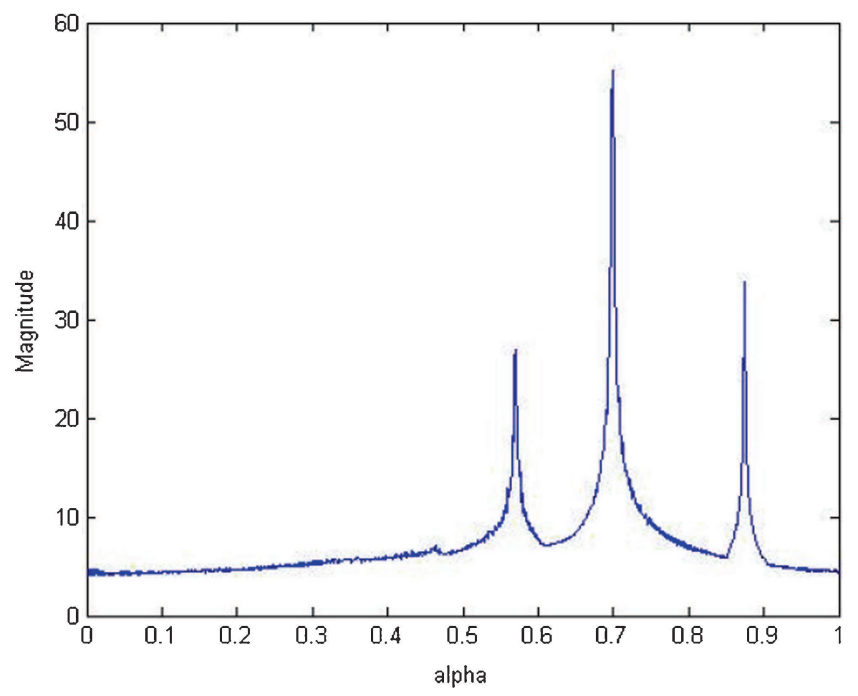

Figure 5. Magnitude vs alpha (low sampling frequency).

If we calculate the chirp characteristics based on the three most prominent peaks we get (50.0139, - 100.5377), (127.9260, 199.6531) and $(200.5458,43.7589)$ which are acceptable given our inputs were $(50,-100),(128,200)$ and $(200,45)$ respectively.

Now if we sample at a higher frequency, the peaks are more prominent and the results are more accurate. Figure 6 shows the estimation for the same signal $y(t)$ at sampling frequency of $2000 \mathrm{~Hz}$. Now only three peaks are visible proving our claim that higher the sampling frequency the easier to estimate the peaks. The calculated chirp characteristics are $(49.9595,-100.4215)$, $(127.9597,199.8093)$ and $(199.7833,45.1816)$, which are more accurate than the estimates at sampling frequency of $1000 \mathrm{~Hz}$. Figure 7 plots the maximum magnitude at an alpha vs alpha for low sampling frequency of $2000 \mathrm{~Hz}$.



Figure 6. Estimation of chirp characteristics in a mixture of non proximally located linear chirps (high sampling frequency). 


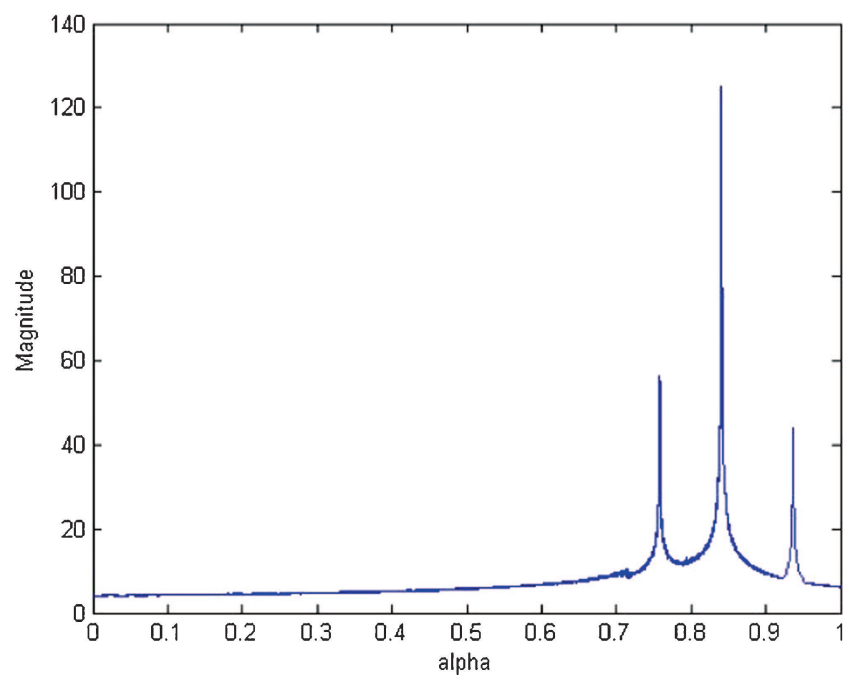

Figure 7. Magnitude vs alpha (high sampling frequency).

7.1b Effect of the stronger chirp in estimating the amplitudes of the chirps in a mixture of LFM chirps: Intuitively if the LFM chirps are located closely in the time-frequency plane i.e. their chirp parameters are close to one another then the interference caused by the stronger one on the others is greater than the effect caused when they are not closely located in the time-frequency plane. The following results support this claim.

Consider the mixed chirp

$$
y=\sin \left(2 \pi\left(50 t^{2}-100 t\right)\right)+k * \sin \left(2 \pi\left(200 t^{2}+200\right)\right) .
$$

This is of the form

$$
y=c_{1} y_{1}+c_{2} y_{2},
$$

where $y_{1}$ and $y_{2}$ are linear chirps and $c_{1}$ and $c_{2}$ are real constants.

Now putting $c_{1}=1$ and varying $c_{2}$, we study the estimates of $c_{1}$ and $c_{2}$, at a fixed sampling frequency $F s$ and sampling time $t$.

Here $F s=6000 \mathrm{~Hz}$ and $t=(0,2) \mathrm{s}$, the sweep of $\alpha$ is $-1: 0.0001:-0.0001$. For the simulations in the table below the chirp parameters estimated for $y_{1}$ and $y_{2}$ are $(49.9698,-100.4399)$ and $(200.0361,199.6908)$ respectively, they are good estimates when compared with the original chirp parameters of $y_{1}$ and $y_{2}$ that are $(50,-100)$ and $(200,200)$ respectively. Table 1 draws the estimates of $c_{1}$ and $c_{2}$ for different input values of $c_{1}$ and $c_{2}$. Figure 8 shows two closely located chirps for which $c_{1}$ and $c_{2}$ are 1 and 1 .

Table 1. LFM chirps close in time-frequency plane.

\begin{tabular}{lccc}
\hline$c_{1}$ & $c_{2}$ & $c_{1 e s t}$ & $c_{2 e s t}$ \\
\hline 1 & 1 & 1.0148 & 1.1262 \\
1 & 2 & 1.0709 & 1.9568 \\
1 & 3 & 1.3850 & 2.8610 \\
1 & 4 & 1.8461 & 3.7395 \\
\hline
\end{tabular}




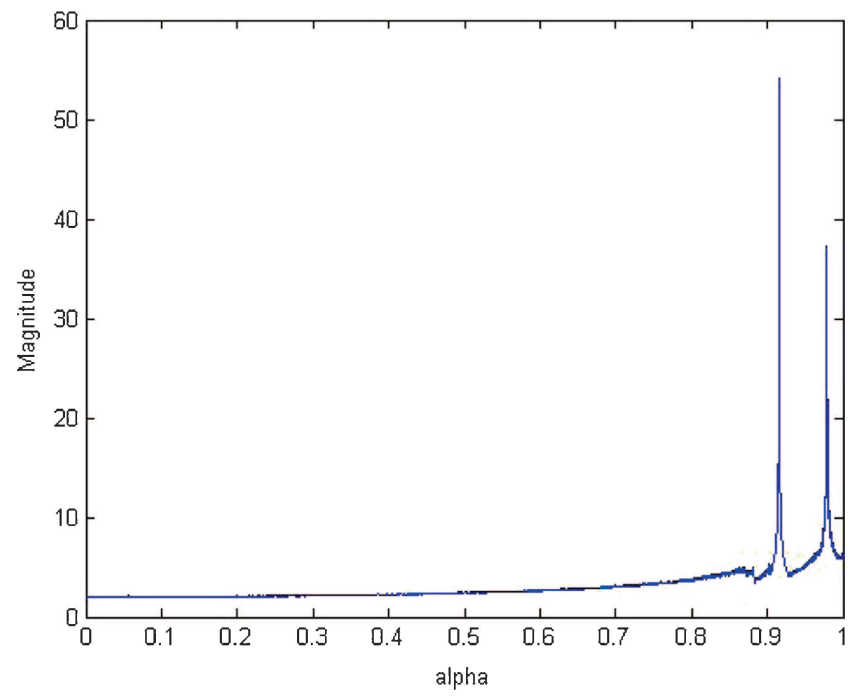

Figure 8. Magnitude vs $\alpha$, for closely located chirps at $\left(c_{1}, c_{2}\right)=(1,1)$.

Notice that if the chirps are closer by (in terms of their chirp rates), the estimates of the amplitudes have huge errors just as their ratio starts getting away from 1, because of the interference from one of them on the other (table 1).

Let us consider chirps that are far away than the previous set and see how the estimates of $c_{1}$ and $c_{2}$ go out to be

$$
y=\sin \left(2 \pi *\left(50 t^{2}-100 t\right)\right)+k * \sin \left(2 \pi\left(400 t^{2}+200\right)\right) .
$$

Now putting $c_{1}=1$ and varying $c_{2}$, we study the estimates of $c_{1}$ and $c_{2}$, at a fixed sampling frequency $F s$ and sampling time $t$.

Here $F s=6000 \mathrm{~Hz}$ and $\mathrm{t}=(0,2)$ s, the sweep of $\alpha$ is $-1: 0.0001:-0.0001$. For the simulations in the table below the chirp parameters estimated for $y_{1}$ and $y_{2}$ are $(49.9698,-100.4399)$ and $(399.9883,199.7783)$ respectively, they are good estimates when compared with the original chirp parameters of $y_{1}$ and $y_{2}$ that are $(50,-100)$ and $(400,200)$ respectively. Table 2 draws the estimates of $c_{1}$ and $c_{2}$ for different input values of $c_{1}$ and $c_{2}$. Figure 9 shows two far located chirps for which $c_{1}$ and $c_{2}$ are 1 and 1 .

We see that if the chirps are far enough in the FrFT domain i.e. if their chirp rates are far away, then the estimates of $c_{1}$ and $c_{2}$ are much more precise than that of the closer chirps (table 2).

Table 2. LFM chirps not closely located in time-frequency plane.

\begin{tabular}{cccc}
\hline$c_{1}$ & $c_{2}$ & $c_{1 \text { est }}$ & $c_{2 \text { est }}$ \\
\hline 1 & 1 & 1.0067 & 1.2172 \\
1 & 2 & 1.0380 & 2.0953 \\
1 & 3 & 1.0896 & 3.0267 \\
1 & 4 & 1.2192 & 3.9860 \\
1 & 5 & 1.5239 & 4.9544 \\
1 & 6 & 1.8287 & 5.9269 \\
\hline
\end{tabular}




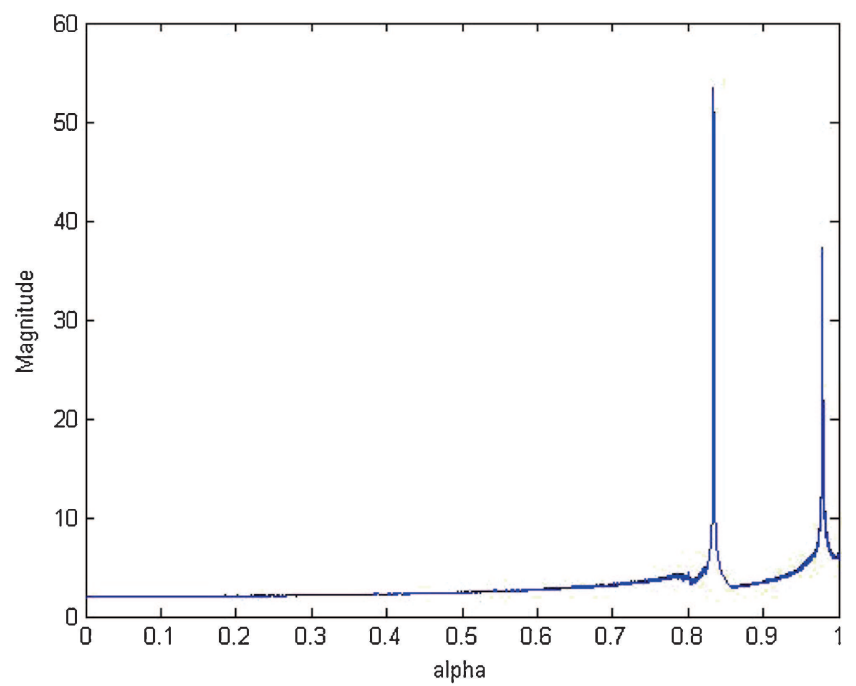

Figure 9. Magnitude vs $\alpha$ for not closely located chirps at $\left(c_{1}, c_{2}\right)=(1,1)$.

\subsection{QFM chirps}

7.2a Identification of single QFM chirp in Gaussian noise: Before separation of multiple chirps, we want to analyse the performance of our method for identification of a single chirp in noise. We consider a single Quadratic chirp sampled at a frequency of $2000 \mathrm{~Hz}$ for $5 \mathrm{~s}$ and corrupted with white Gaussian noise. We apply the GTFT to this signal and search for peaks in the transform. The signal has been correctly identified if the location of the global maxima in this transform gives the correct values of the chirp parameters. This simulation was done multiple times for SNR varying from $-10 \mathrm{~dB}$ to $-30 \mathrm{~dB}$. At an SNR of $-25 \mathrm{~dB}$, we found that the chirp was not correctly identified in 1 out of 10 simulations. At an SNR of $-24 \mathrm{~dB}$, the chirp was correctly identified in all 10 simulations. Figure 10 shows the GTFT of one such noise corrupted signal with and SNR of $-24 \mathrm{~dB}$. The method also worked perfectly for all simulations with a higher SNR.

The chirp under consideration is

$$
y=e^{i\left(20 t^{3}+2 \pi\left(60 t^{2}+37 t\right)\right)}+n(t),
$$

where $n(t)$ is an Additive White Gaussian Noise with SNR $=-24 \mathrm{db}$. Here $F s=2000, t=$ $(0,5)$, the sweep of $\mathrm{p}$ is 19:0.1:21 and the sweep of alpha is $-1: 0.001:-0.001$. The estimated values were $(19.9,60.1611,36.4514)$ as opposed to our considered $(20,60,37)$ which indicate satisifactory results.

We can claim that the method works reasonably well for all chirps with an SNR greater than $-24 \mathrm{~dB}$. This performance can be improved even further by analysing the signal for a longer time. While the number of simulations is not sufficient to determine this threshold with certainty, it does give us a fair idea about the performance of this method. This method seems to be highly resistant to noise just like the FRFT. In practice, we are unlikely to deal with signals with such high noise. Therefore, our further study will focus on just the application of this method to identify all the chirps in a mixture of chirps without noise. 


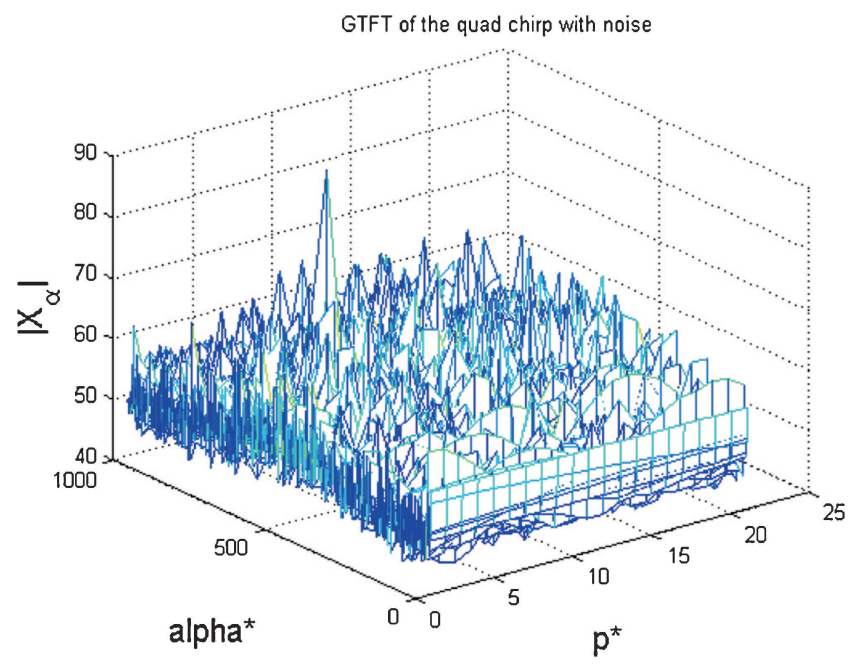

Figure 10. Estimation of Quadratic chirp under noise of SNR $=-24 \mathrm{db}$, plot of the maxima of GTFT against varying $\alpha$ and $\psi$.

7.2b Identification of chirp parameters in a mixture of QFM chirps: Consider the signal

$$
y=\sin \left(20 t^{3}+2 \pi *\left(60 t^{2}+37 t\right)\right)+\sin \left(70 t^{3}+2 \pi *\left(200 t^{2}+100 t\right)\right),
$$

where $c_{1}(t)=\sin \left(20 t^{3}+2 \pi *\left(60 t^{2}+37 t\right)\right)$, and $c_{2}(t)=\sin \left(70 t^{3}+2 \pi *\left(200 t^{2}+100 t\right)\right)$.

The results were satisfactory with the parameters being estimated as $(19,61.4002,32.9760)$ against the actual values $20,60,37$ for the first chirp and $(70,200.1287,99.3515)$ against $(70,200,100)$ for the second chirp (figure 11).

Consider the mixture of chirps $y=y_{1}(t)+y_{2}(t)$, where $y_{1}(t)=\sin \left(a_{1} t^{3}+2 \pi *\left(b_{1} t^{2}+c_{1} t\right)\right)$ is Chirp 1 with parameters $\left(a_{1}, b_{1}, c_{1}\right)$, similarly for $y_{2}(t)$. Table 3 shows the simulation results for different values of the parameters of $y_{1}(t)$ and $y_{2}(t)$, estimated from $y(t)$. For all the simulations

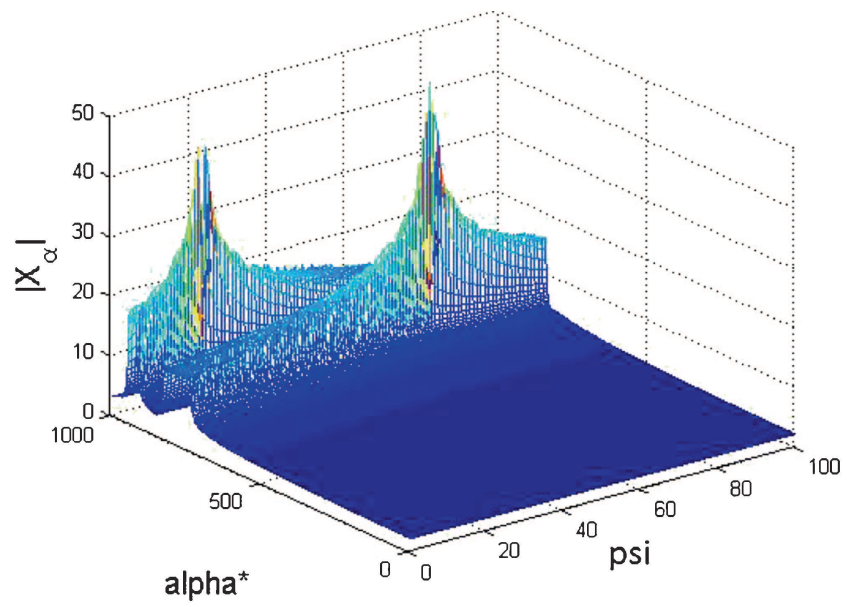

Figure 11. Estimation of Quadratic chirps, plot of the maxima of GTFT against varying $\alpha$ and $\psi$. 
Table 3. Estimation of individual Quadratic Chirp parameters from a mixture of two QFM chirps.

\begin{tabular}{lccc}
\hline Chirp 1 Parameters & Chirp 2 Parameters & Est Chirp 1 Parameters & Est Chirp 2 Parameters \\
\hline$(20,50,-100)$ & $(70,200,200)$ & $(19,50.7375,-99.0794)$ & $(71,198.8672,202.3124)$ \\
$(20,50,-100)$ & $(25,200,200)$ & $(19,50.7375,-100.0818)$ & $(26,198.8672,202.3124)$ \\
$(40,30,-100)$ & $(50,80,200)$ & $(39,30.6373,-108.0538)$ & $(50,80.1935,208.5765)$ \\
$(40,55,-100)$ & $(62,68,200)$ & $(41,54.2206,-151.3025)$ & $(61,68.3826,204.5652)$ \\
\hline
\end{tabular}

in table $3, F s=6000$, the sweep of $a$ (co-efficient of $t^{3}$ in the phase) is 1:1:100, the sweep of $\alpha$ is $-0.001: 0.001:-1$. For the simulations in rows 1 and 2 , time is $(0,4) \mathrm{s}$, for the simulations in rows 3 and 4 , time is $(0,2) \mathrm{s}$.

Note: A linear chirp is essentially a QFM chirp with the quadratic chirp rate parameter set to 0 . So a mixture of LFM chirps and QFM chirps can be treated as a mixture of QFM chirps and the GTFT can be applied to characterize individual chirps.

\section{Uncertainty principle for the General Time-Frequency Transform}

The uncertainty principle in the time-frequency plane plays an important role in signal processing. In Shinde \& Gadre (2001), the uncertainty principle has been derived for a signal in the time and FRFT domain. We try to derive a similar uncertainty principle for a signal in the time and General Time-Frequency Transform(GTFT) domain.

The kernel of the GTFT is

$$
K_{\phi}(x, y)=K_{0}(\phi) e^{j\left(h_{1}(y, \phi)-K_{1}(\phi) x y+h_{2}(x, \phi)\right)} .
$$

The GTFT looks like

$$
\begin{gathered}
G_{\phi}(f, u)=K_{0} \int f(t) e^{j\left(h_{1}(u, \phi)\right)} e^{-j\left(K_{1}(\phi) u t\right)} e^{j\left(h_{2}(t, \phi)\right)} d t \\
=K_{0} e^{j\left(h_{1}(u, \phi)\right)} \int f(t) e^{-j\left(K_{1}(\phi) u t\right)} e^{j\left(h_{2}(t, \phi)\right)} d t \\
\tilde{f}(t)=f(t) e^{j\left(h_{2}(t, \phi)\right)} \\
|\tilde{f}(t)|=|f(t)| .
\end{gathered}
$$

From Eq. (54) and Eq. (55)

$$
\left|G_{\phi}(f, u)\right|=\left|K_{0}(\phi)\right| . \tilde{F}\left(K_{1}(\phi)(u)\right) .
$$

From Eq. (56)

$$
\begin{aligned}
& \int\left|G_{\phi}(f, u)\right|^{2}=\left|K_{0}(\phi)\right|^{2} \int\left|\tilde{F}\left(K_{1}(\phi)(u)\right)\right|^{2} d u \\
& =\frac{\left|K_{0}(\phi)\right|^{2}}{\left|K_{1}(\phi)\right|^{2}} \int\left|\tilde{F}\left(K_{1}(\phi)(u)\right)\right|^{2} d\left(K_{1}(\phi) u\right) \\
& =\frac{\left|K_{0}(\phi)\right|^{2}}{\left|K_{1}(\phi)\right|} \int|\tilde{F}(v)|^{2} d v \\
& =\frac{\left|K_{0}(\phi)\right|^{2}}{\left|K_{1}(\phi)\right|} \int|F(v)|^{2} d v
\end{aligned}
$$

And

$$
\begin{aligned}
& \int u^{2}\left|G_{\phi}(f, u)\right|^{2} d u \\
& =\int u^{2}\left|K_{0}(\phi)\right|^{2} \mid \tilde{F}\left(\left.K_{1}(\phi)(u)\right|^{2} d u\right. \\
& =\frac{\left|K_{0}(\phi)\right|^{2}}{\left|K_{1}(\phi)\right|^{3}} \int v^{2}|\tilde{F}(v)|^{2} d v .
\end{aligned}
$$


Uncertainty for $\tilde{f}(t)$

$$
\frac{\int t^{2}|\tilde{f}(t)|^{2} d t \int u^{2}|\tilde{F}(u)|^{2} d u}{\int|\tilde{f}(t)|^{2} d t \int|\tilde{F}(u)|^{2} d u} \geq \frac{1}{4} .
$$

From Eq. (55), Eq. (57) and Eq. (58)

$$
\begin{aligned}
& \frac{\int t^{2}|f(t)|^{2} d t}{\int|f(t)|^{2} d t} \cdot \frac{\int u^{2}\left|G_{\phi}(f, u)\right|^{2} d u \cdot \frac{\left|K_{1}(\phi)\right|^{3}}{\left|K_{0}(\phi)\right|^{2}}}{\int\left|G_{\phi}(f, u)\right|^{2} d u \cdot \frac{\left|K_{1}(\phi)\right|}{\left|K_{0}(\phi)\right|^{2}}} \geq \frac{1}{4} \\
& =\frac{\int t^{2}|f(t)|^{2} d t}{\int|f(t)|^{2} d t} \cdot \frac{\int u^{2}\left|G_{\phi}(f, u)\right|^{2} d u}{\int\left|G_{\phi}(f, u)\right|^{2} d u} \geq \frac{1}{4\left|K_{1}(\phi)\right|^{2}} .
\end{aligned}
$$

Hence the uncertainty principle for the GTFT can be written as

$$
\frac{\int t^{2}|f(t)|^{2} d t}{\int|f(t)|^{2} d t} \cdot \frac{\int u^{2}\left|G_{\phi}(f, u)\right|^{2} d u}{\int\left|G_{\phi}(f, u)\right|^{2} d u} \geq \frac{1}{4\left|K_{1}(\phi)\right|^{2}} .
$$

This formula limits the spread of the signal in the time and GTFT domain.

Note: For the conventional FrFT, $K_{1}(\phi)=\csc \phi$.

So the bound becomes $\left(\frac{1}{4} \sin ^{2} \phi\right)$ which is consistent with the result obtained in Shinde \& Gadre (2001) for real signals.

\section{Conclusion and suggested further scope of work}

Two methods have been proposed for parameter estimation of IF of constant amplitude chirps in a mixture of multiple chirps - separately for mixture of LFM chirps and for mixture of QFM chirps. The encouraging results for a mixture of chirps, which are very closely located on the time-frequency plane shows the strength of the GTFT and this method. As mentioned, for the simulations, correlation between the time-frequency curves of the two chirps in the mixture was chosen to be in the range $(0.99,0.999)$. The methods of spectral-estimation and chirp-separation during the estimation procedure are novel and very effective.

Suggested further scope of work includes utilization of the GTFT for parameter estimation of chirps having an esoteric relation of IF with time. This can be investigated by exploiting the freedom provided in choosing $f(\cdot)$ in the expression for GTFT given at Eq. (28). The uncertainty principle for the GTFT may have some interesting implications in estimating QFM signals and higher order chirps, which need to be studied.

\section{A. Appendix}

\section{A.1 Proof of time shift property in GTFT}

Suppose $Y_{\alpha}(u)$ is the GTFT of $y(t)$ at angle $\alpha$ with $f(t)=k t^{3}$, for a constant $k$,

$$
\text { i.e. } Y_{\alpha}(u)=\int_{-\infty}^{\infty} y(t) K_{\alpha, f}(u, t) \mathrm{d} t
$$


Our aim is to find the GTFT of $y^{1}(t)=y\left(t-t_{0}\right)$ at same angle and with same function $f(t)$.

$$
\begin{aligned}
Y_{\alpha}^{1}(u) & =\int_{-\infty}^{\infty} y\left(t-t_{0}\right) K_{\alpha, f}(u, t) \mathrm{d} t \\
& =\int_{-\infty}^{\infty} y\left(t-t_{0}\right) K_{\alpha, f}(u, t) \mathrm{d} t \\
& =\sqrt{\frac{1-j \cot \alpha}{2 \pi}} \int_{-\infty}^{\infty} y\left(t-t_{0}\right) e^{j\left[\frac{u^{2}}{2} \cot (\alpha)+\frac{t^{2}}{2} \cot (\alpha)-u t \csc (\alpha)+k u^{3}-k t^{3}\right]} \mathrm{d} t .
\end{aligned}
$$

Substitute $t=t_{0}+\tau$

$$
Y_{\alpha}^{1}(u)=\sqrt{\frac{1-j \cot \alpha}{2 \pi}} \int_{-\infty}^{\infty} y(\tau) e^{j\left[\frac{u^{2}}{2} \cot (\alpha)+\frac{\left(\tau+t_{0}\right)^{2}}{2} \cot (\alpha)-u\left(\tau+t_{0}\right) \csc (\alpha)+k u^{3}-k\left(\tau+t_{0}\right)^{3}\right]} \mathrm{d} \tau .
$$

Consider two new variables-

$$
\begin{gathered}
\cot \beta=\cot \alpha-6 k t_{0} . \\
\text { and } \quad \mu=\sin \beta\left(u \csc \alpha+3 k t_{0}^{2}-t_{0} \cot \alpha\right)
\end{gathered}
$$

Substituting these variables in (5), we get,

$$
\begin{aligned}
& Y_{\alpha}^{1}(u)=\sqrt{\frac{1-j \cot \alpha}{2 \pi}} \int_{-\infty}^{\infty} y(\tau) e^{j\left[\frac{\mu^{2}}{2} \cot (\beta)+\frac{\tau^{2}}{2} \cot (\beta)-\mu \tau \csc (\beta)+k \mu^{3}-k \tau^{3}\right]} \\
& \cdot e^{j\left[\frac{u^{2}}{2} \cot \alpha+k u^{3}-u t_{0} \csc \alpha+\frac{t_{0}^{2}}{2} \cot \alpha-k t_{0}^{3}-\frac{\mu^{2}}{2} \cot \beta-k \mu^{3}\right]} \mathrm{d} \tau
\end{aligned}
$$

But, by definition

$$
K_{\beta, f}(\mu, \tau)=\sqrt{\frac{1-j \cot \alpha}{2 \pi}} e^{j\left[\frac{\mu^{2}}{2} \cot (\beta)+\frac{\tau^{2}}{2} \cot (\beta)-\mu \tau \csc (\beta)+k \mu^{3}-k \tau^{3}\right]} .
$$

Therefore,

$$
\begin{aligned}
Y_{\alpha}^{1}(u) & =\int_{-\infty}^{\infty} y(\tau) K_{\beta, f}(\mu, \tau) e^{j\left[\frac{u^{2}}{2} \cot \alpha+k u^{3}-u t_{0} \csc \alpha+\frac{t_{0}^{2}}{2} \cot \alpha-k t_{0}^{3}-\frac{\mu^{2}}{2} \cot \beta-k \mu^{3}\right]} \mathrm{d} \tau \\
& =e^{j\left[\frac{u^{2}}{2} \cot \alpha+k u^{3}-u t_{0} \csc \alpha+\frac{t_{0}^{2}}{2} \cot \alpha-k t_{0}^{3}-\frac{\mu^{2}}{2} \cot \beta-k \mu^{3}\right]} \int_{-\infty}^{\infty} y(\tau) K_{\beta, f}(\mu, \tau) \mathrm{d} \tau \\
& =e^{j\left[\frac{u^{2}}{2} \cot \alpha+k u^{3}-u t_{0} \csc \alpha+\frac{t_{0}^{2}}{2} \cot \alpha-k t_{0}^{3}-\frac{\mu^{2}}{2} \cot \beta-k \mu^{3}\right]} Y_{\beta}(\mu) .
\end{aligned}
$$

This is the time shift property of the GTFT.

\section{References}

Almeida L B 1994 The fractional Fourier transform and time-frequency representations. IEEE Trans. Signal Process. 42: 3084-3091

Bultheel A and Martinez H 2003 A shattered survey of the Fractional Fourier Transform

Cai L 2000 Special affine Fourier transformation in frequency-domain. Optics Commun. 185: 271-276

Chassande-Mottin E and Flandrin P 1999 On the time frequency detection of chirps. Appl. Comput. Harmonic Anal. 6: 252-281

Djuric P M and Kay S M 1990 Parameter estimation of chirp signals. IEEE Trans. Acoutics Speech Signal Process. 38: 12

Gal J, Campeanu A and Nafornita I 2011 The estimation of chirp signals parameters by an extended Kalman filtering algorithm. In: Signals, Circuits and Systems (ISSCS), 2011 10th International Symposium on, vol., no., pp. 1-4 
Ma Min, Cai J and Zhang X 2010 An estimation algorithm of chirp based on fractional fourier transform and frequency domain differential. In: Computational Problem-Solving (ICCP), 2010 International Conference on, vol., no., pp. 218-221

Namias V 1980 The Fractional Fourier transform and its application to quantum mechanics. J. Inst. Math. Appl. 25: 241-265

Ozaktas H, Arikan O, Kutay A and Bozdagi G 1996 Digital computation of the fractional fourier transform. IEEE Trans. Signal Process. 44: 9

Roshen Jacob, Tessamma Thomas and Unnikrishnan A 2009 Applications of Fractional Fourier Transform in sonar signal processing. IETE J. Res. 55: 16-27

Shinde Sudarshan and Gadre Vikram 2001 An uncertainty principle for real signals in the Fractional Fourier transform domain. IEEE Trans. Signal Process. 49: 11

Shishir Sahay, Deepak Pande, Vikram Gadre and Prashant Sohani 2012a A novel generalized timefrequency transform inspired by the Fractional Fourier Transform for higher order chirps. In: International Conference on Signal Processing (SPCOM)

Sahay, Pande D, Vikram Gadre and Prashant Sohani 2012b Model-independent approach for chirp parameter estimation employing collection of filters. In: National Conference on Communications (NCC) http://www.mathworks.in/matlabcentral/fileexchange/41351-frft-m/content/frft.m 